\title{
PROYECTOS FORESTALES DE MECANISMO DE DESARROLLO LIMPIO EN COLOMBIA: UNA MIRADA DESDE EL DESARROLLO SOSTENIBLE LOCAL ${ }^{*}$
}

\author{
JAVIER SABOGAL AGUILAR"* \& EDGAR MORENO CASTILLO"** \\ UNIVERSIDAD MILITAR NUEVA GRANADA
}

Recibido/Received/Recebido: 10/10/2011 - Aceptado/ Accepted/Aprovado: 19/04/2011

\begin{abstract}
Resumen
La investigación evaluó dos proyectos forestales colombianos de Mecanismo de Desarrollo Limpio para analizar los beneficios aportados al desarrollo sostenible local en cada caso. Se comparó el uso del suelo para ganadería extensiva, como uso más probable en ausencia de desarrollo de proyectos alternativos, versus el establecimiento de plantaciones forestales. Para ello, se realizó una evaluación multicriterio con líderes de grupos de interés de orden local ligados a los dos casos de estudio (Cuenca del río Chinchiná - PROCUENCA y Captura de Carbono y Agroforestería del Valle de San Nicolás). Este estudio, permitió concluir que estos proyectos han contribuido al desarrollo sostenible local de áreas rurales dado que fomentaron el desarrollo del potencial forestal, generaron conocimientos valiosos en gestión ambiental y su ejecución tuvo en cuenta múltiples actores locales. No obstante, se identificaron necesidades técnicas, de participación y organización local para lograr aportes efectivos.
\end{abstract}

Palabras clave: Desarrollo sostenible local, Mecanismo de desarrollo limpio (MDL), proyectos forestales, servicios ambientales.

\section{FORESTRY PROJECTS OF CLEAN DEVELOPMENT MECHANISM IN COLOMBIA: AN OVERVIEW FROM LOCAL SUSTAINABLE DEVELOPMENT}

\begin{abstract}
This research assessed two Colombian forestry projects of Clean Development Mechanism to analyze the benefits to local sustainable development in each case. Land use was compared for extensive livestock, as most likely use in absence of alternative development projects versus the establishment of forestry plantations. For this purpose, a multi-criterion assessment was made with local interest group leaders linked to both case studies (Chinchiná River Basin - PROCUENCA and Carbon capture and agroforestry of San Nicholas valley). This study concludes that these projects have contributed to local sustainable development of rural areas given that they fomented potential
\end{abstract}

Artículo resultado del proyecto ECO 569 de la Facultad de Ciencias Económicas, Universidad Militar Nueva Granada. Con financiación de la Vicerrectoría de Investigaciones Universidad Militar Nueva Granada.

* Docente Investigador Universidad Militar Nueva Granada, Facultad de Ciencias Económicas. Doctor (c) en Ciencias Económicas de la Universidad Nacional de Colombia, Magíster en Medio Ambiente y Desarrollo: Gestión Ambiental y Administrador de Empresas de la misma universidad. E-mail: javier.sabogal@unimilitar.edu.co

*** Investigador Universidad Militar Nueva Granada, Facultad de Ciencias Económicas. Magíster en Medio Ambiente y Desarrollo y Biólogo de la Universidad Nacional de Colombia. E-mail: edgar.moreno@unimilitar.edu.co 
forestry development, they generated valuable knowledge in environmental management and their execution considered multiple local actors. Notwithstanding, participation and local organizational technical needs were identified to achieve effective contributions.

Keywords: Local sustainable development, clean development mechanism (CDM), forestry projects, environmental services.

\title{
PROJETOS FLORESTAIS DE MECANISMO DE DESENVOLVIMENTO LIMPO NA COLÔMBIA: UMA OLHADA DESDE O DESENVOLVIMENTO SUSTENTÁVEL LOCAL
}

\begin{abstract}
Resumo
A pesquisa avaliou dois projetos florestais colombianos de MDL para analisar os benefícios proporcionados ao desenvolvimento sustentável local em cada caso. Comparou-se o uso da terra para a pecuária, uso mais provável na ausência de projetos de desenvolvimento alternativo, frente aos cultivos florestais. Para este fim, realizou-se uma avaliação de vários critérios com os líderes de grupos locais de interesse relacionados aos dois casos de estudo (Bacia do rio Chinchiná - PROCUENCA - e Captura de Carbono Agroforestería do Val de San Nicolás). O estudo concluiu que esses projetos têm contribuído para o desenvolvimento local sustentável em áreas rurais porque incentivam o desenvolvimento do potencial florestal, geraram um conhecimento valioso na gestão ambiental e levaram em conta muitos atores locais na sua execução. No entanto, identificaram-se necessidades técnicas, assim como de participação e organização local para fazer contribuições efetivas.
\end{abstract}

Palavras chave: Desenvolvimento sustentável local, mecanismo de desenvolvimento limpo (MDL), projetos florestais, serviços ambientais.

Sabogal, J. \& Moreno, E. (2011). Proyectos forestales de mecanismo de desarrollo limpio en Colombia: una mirada desde el desarrollo sostenible local. En: Revista de la Facultad de Ciencias Económicas de la Universidad Militar Nueva Granada. rev.fac.cienc.econ, XIX (1).

JEL: O22, O44, Q23, Q20.

\section{Introducción}

La problemática ambiental global relacionada con el Cambio Climático, ha sido generada por desequilibrios en los sistemas de interrelaciones sociedadnaturaleza producto de actividades de producción y consumo; este hecho marca la necesidad de aceptación de responsabilidades que deben asumir de manera diferenciada las naciones y sectores de acuerdo con su contribución histórica al problema y a su capacidad de adaptación y mitigación.

Partiendo del principio de responsabilidades compartidas pero diferenciadas y de la necesidad de acciones en el menor tiempo posible, debido al aumento exponencial de los costos proporcional a la demora en la toma de decisiones (Stern, 2007), se plantea la arquitectura internacional del Protocolo de Kioto, que incluyó al Mercado de Carbono como instrumento costo efectivo que involucra las diferencias que existen entre países en el costo marginal de disminución de gases efecto invernadero (GEI). Es así como aparecen los mecanismos de flexibilización y dentro de ellos, el Mecanismo de Desarrollo Limpio (MDL) que involucra la participación de países industrializados con metas de reducción de emisiones ante el Protocolo de Kioto (países Anexo I) y países en desarrollo sin compromisos de 
reducción (países no Anexo I). Respecto al mencionado mecanismo, los planteamientos institucionales promulgan que los países Anexo I ganan el cumplir con sus compromisos de manera costo efectiva y los países no Anexo I desarrollan proyectos de MDL que contribuyen a las metas en desarrollo sostenible (United Nations Framework Convention on Climate Change, 1997).

Entre los proyectos forestales, las actividades de forestación y reforestación por tratarse de actividades de carácter temporal o transitorio, siguen siendo las únicas medidas aceptadas para proyectos MDL (United Nations Framework Convention on Climate Change, 2002; United Nations Framework Convention on Climate Change, 2004). Estos proyectos forestales permiten alcanzar una forma más sostenible de desarrollo en países no Anexo I, en la medida en que mejoran índices sociales, económicos, de salud y generan beneficios conexos a la actividad forestal y al establecimiento de ecosistemas forestales, como son la mitigación de efectos de sequías y la conservación de la biodiversidad, entre otros (Teixeira, Murray \& Carvalho, 2006).

Dada la propuesta institucional anteriormente descrita, se requiere hacer un análisis del aporte al desarrollo sostenible de estos proyectos, y comprobar que las promesas institucionales se cumplen en los procesos que se están desarrollando. De manera particular, se plantea evaluar la contribución a escala local de proyectos que involucren actividades forestales, por su intervención directa sobre el medio, sobre el paisaje y sobre el uso y manejo de los recursos naturales. De igual manera, se hace necesario analizar las relaciones entre los grupos de interés y las empresas responsables de la ejecución e intermediación de los proyectos, dados los compromisos a nivel local en torno a las estrategias de desarrollo sostenible y de gestión ambiental, que deben ser evaluadas para dimensionar el alcance de este tipo de proyectos en la sostenibilidad local.

De esta manera, se aborda el aporte al desarrollo sostenible a nivel local en los proyectos forestales "Cuenca del río Chinchiná - PROCUENCA" y "Captura de Carbono y Agroforestería del Valle de San Nicolás", enmarcados en el Mecanismo de
Desarrollo Limpio, desde la construcción conceptual de: ambiente, desarrollo sostenible local y complejidad en sistemas sociales, tomando en cuenta la evolución de procesos: territoriales, ambientales, biofísicos, socio-culturales y económicos, relacionados con el mejoramiento /empeoramiento de las condiciones de vida de poblaciones humanas a nivel local. Lo anterior enmarcado en las políticas y programas de contribución al desarrollo sostenible y gestión ambiental propuestas en los proyectos.

\section{Una mirada ambiental desde el desarrollo sostenible local}

La problemática analizada en el presente estudio se aborda desde su condición ambiental y parte de la visión de ambiente planteada por Carrizosa como concepto:
"Que relaciona ecosistemas y culturas para el análisis de procesos", viendo así al ambiente como algo más que recursos (Carrizosa, 2000, 15).

Este mismo autor señala que en torno a la concepción de ambiente se han conformado tres grandes conjuntos de elementos o componentes ambientales: lo natural no antrópico, lo construido o transformado y lo sociocultural, cuya sumatoria constituye la realidad ambiental. Para abarcar la complejidad de este análisis y la operatividad de la gestión ambiental al definir el ambiente como grupos de interrelaciones, se parte de la definición sistémica de ambiente, entendiéndolo como un sistema en otro sistema interrelacionado (Carrizosa, 2000). Este enfoque requiere el esfuerzo de una mirada transdisciplinar que pueda ser llevada al terreno de la gestión, propuesta que supone el reto de penetrar y abrirse campo entre la lógica económica y financiera, dominantes en nuestro mundo, que opone las metas ambientales a las económicas como realidad dicotómica.

En este orden de ideas, la aproximación reduccionista a lo ambiental se intensifica como consecuencia de la oposición entre metas ambientales y económicas, con clara dominación del papel de lo económi- 
co, respecto del cual se acepta como tendencia general, el crecimiento económico como acepción del desarrollo, lo que está profundamente arraigado en nuestra sociedad. Es claro que ésta no es una conclusión sin discusión, por el contrario, el concepto de desarrollo como se interpreta en la actualidad es reciente y resultado del desenvolvimiento histórico de la geopolítica global, y prima por ser dominante en los ámbitos de toma de decisiones y entre los hacedores de política (Sabogal \& Hurtado, 2009); y esta preponderancia se manifiesta precisamente, en la errónea aplicación de modelos económicos muy específicos para la solución de problemas ambientales complejos (Carrizosa, 2005).

Sabogal \& Hurtado (2009) muestran que desde esta óptica economicista existe un conflicto entre ambiente y desarrollo que se ha planteado de manera dicotómica, sobre todo en las relaciones entre naciones industrializadas y en desarrollo, o relaciones Norte-Sur, situación ante la cual las salidas que usualmente se proponen son de corte tecnológico, desconociendo el carácter social de los problemas ambientales. Esta tendencia de dar solución a los problemas ambientales de cara al desarrollo a través de la tecnología y la retribución económica, está presente en la propuesta de mitigación del cambio climático del Protocolo de Kioto y en las negociaciones del siguiente acuerdo multilateral que lo reemplazará en años posteriores al 2012 (Olsen \& Fenhann, 2008a; Keeler, 2008), al incluir la transferencia tecnológica Norte-Sur como la forma de aporte a la sostenibilidad de los "países en desarrollo", lo que se ve en los tipos de proyectos que componen el MDL. La alternativa a esta visión reduccionista por tanto, debe basarse en la aceptación de la complejidad involucrada en los temas ambientales y el asumir una racionalidad ambiental como alternativa a la económica-tecnológica (Sabogal, 2008).

\subsection{Desarrollo Sostenible y su relación con lo local}

La sostenibilidad global se evalúa como producto del total de interacciones al interior de un territorio, modelo o sistema, lo que facilita el proceso, pero deja a un lado la complejidad de las relaciones y los valores éticos y de justicia (Carrizosa, 2005), de manera que al analizar el desarrollo sostenible se hace necesario involucrar la escala a la cual se desea ver la sostenibilidad.

Así, un análisis alternativo es la escala local que debe tener en cuenta la delimitación de áreas de estudio y abordar las particularidades del territorio.

El desarrollo sostenible alude entonces, al proceso que asegura para un grupo poblacional, sociedad o comunidad, lograr el mejoramiento de sus condiciones de vida mientras mantiene las condiciones biofísicas y ecológicas críticas que son esenciales para la sobrevivencia colectiva. En este sentido, el abordaje alternativo viene soportado en la planificación biorregional (Guimarâes, 2001) y la visión de desarrollo a diferentes escalas territoriales; asumiendo la desigualdad en el desempeño de los territorios y acciones que abarcan: lo social, lo ambiental y lo económico (Borja \& Castells, 1998; Carmona Maya, 2001; Carrizosa, 2001; Clark, 1995; Escobar, 1996; Moncayo, 2001; Sagar, 2000).

El presente artículo aborda la contribución a la sostenibilidad en la escala local, basado en la definición de desarrollo sostenible local de Carrizosa (2005), como aquel desarrollo que es:

"Dependiente de las particularidades del lugar, se sujeta a las condiciones, los cambios y la creatividad para el uso del territorio por sus habitantes, está definida a través de procesos, se hace visible en la interacción de la sostenibilidad biofísica con la sostenibilidad cultural del lugar, incorpora la participación de todos los estamentos de la sociedad y el alcance de las decisiones políticas, atendiendo a valores éticos de equidad intra - e inter-generacional" (Carrizosa, 2005, 36).

Así el desarrollo que se plantea desde el concepto de territorio, toma como referencia uno o más procesos y su evolución en el tiempo y en cada territorio puede caracterizarse por su capacidad de sostener un proceso dado. 


\subsection{Estudio del Desarrollo Sostenible Local en Proyectos Forestales MDL}

Frente a las promesas que genera la visión institucional generalmente aceptada, en cuanto a la contribución del MDL al cumplimiento de sus dos objetivos constitutivos y a la sostenibilidad, están los resultados generados por la investigación teórica y los estudios de caso. En este sentido, se retoman los estudios que han evidenciado que China e India, como los mayores anfitriones de proyectos MDL, han creado políticas que incentivan bajos niveles de sostenibilidad con el fin de atraer más proyectos a menores costos, lo que se ha denominado "race to the bottom" (Jamal, 2006; Sutter \& Parreño, 2007). Lo anterior es preocupante dado que se deja de lado el criterio de contribución al desarrollo sostenible de tales proyectos.

En el caso de los proyectos de aforestación y reforestación dentro del MDL, los beneficios que están dejando a los países anfitriones generan preocupación para los países en desarrollo. La falta de resultados en metas de sostenibilidad en comunidades o poblaciones involucradas en estos proyectos y el que los flujos financieros se concentren en pocos sectores y países, da lugar a una evaluación de la contribución real de los proyectos (Brown, Adger, Boyd, Corbera \& Shackley, 2004; Hepburn, 2007; Olsen \& Fenhann, 2008b; Olsen \& Fenhann, 2008a; Sutter, 2003; Sutter \& Parreño, 2007).

Los beneficios que se establecen para las comunidades locales también son diversos, algunos sólo generan beneficios hasta que las plantaciones alcanzan su madurez, mientras que otros incluyen beneficios en el corto y mediano plazo, por fortalecimiento de instituciones locales que: aumentan el capital social, capacitan en el manejo de bosques y generan empleo (Grieg-Gran, Porras, \& Wunder, 2005; Landell-Mills \& Porras, 2002). Además, para el establecimiento de estos proyectos la inversión externa incentiva y complementa la inversión local y en esta medida, ayuda a enfrentar los requerimientos complejos de registro y certificación (Grieg-Gran et al., 2005).
Dado el debate alrededor del MDL y su contribución al desarrollo sostenible local, se hace necesario someter estos procesos a modelos de evaluación que permitan estimar la sostenibilidad y los impactos de dichos proyectos. Se presenta a continuación un análisis de los criterios utilizados para la evaluación del aporte al desarrollo sostenible y los beneficios derivados de la implementación y ejecución de proyectos de reducción de emisiones de gases efecto invernadero en el marco del Mecanismo de Desarrollo Limpio.

\section{Metodología}

Para abordar la contribución de los proyectos de MDL a la sostenibilidad local fue necesario, una vez definido conceptual y teóricamente el tema, desarrollar metodológicamente criterios de evaluación, adquisición y manejo de información.

Con este fin, se caracterizaron las zonas de influencia de los proyectos bajo estudio, se definieron criterios de análisis de desarrollo sostenible y se llevó a cabo la evaluación de propuestas en desarrollo sostenible de los proyectos examinados. En la definición de estos criterios se retoma a May et al. (2004), quienes proponen una serie de criterios e indicadores que buscan ser una guía para la discusión entre grupos de interés e instituciones, con el fin de incorporar sus preocupaciones de desarrollo sostenible. Estos criterios incluyen componentes: sociales, ambientales y económicos y su aplicación metodológica requiere la identificación y participación de grupos de interés en el orden local y gubernamental (Teixeira et al., 2006).

La metodología usada fue el Análisis de Decisión Multi-criterio (MCDA), la ilustración 1 esquematiza su aplicación, cuyo diseño partió de estudios previos a nivel mundial (De Montis, De Toro, Droste, Omann \& Stagl, 2000; Echeverri, Naranjo \& Uribe, 2003; Fan, Liu \& Tzeng, 2007; Kenyon, 2007; Martinez-Alier, Munda \& O'Neill, 1998; Munda, 2009; Munda, 2004a; Munda, 1993; van der Bergh, 2000). Adicionalmente, se incluyeron recomendaciones propuestas por Munda (Munda, 
2004b; Munda, 2004a) asociadas a incluir una fase de análisis previa, denominada por el autor como análisis institucional, que hace referencia al desarrollo de una línea base que determina insumos importantes para dar solidez al estudio.

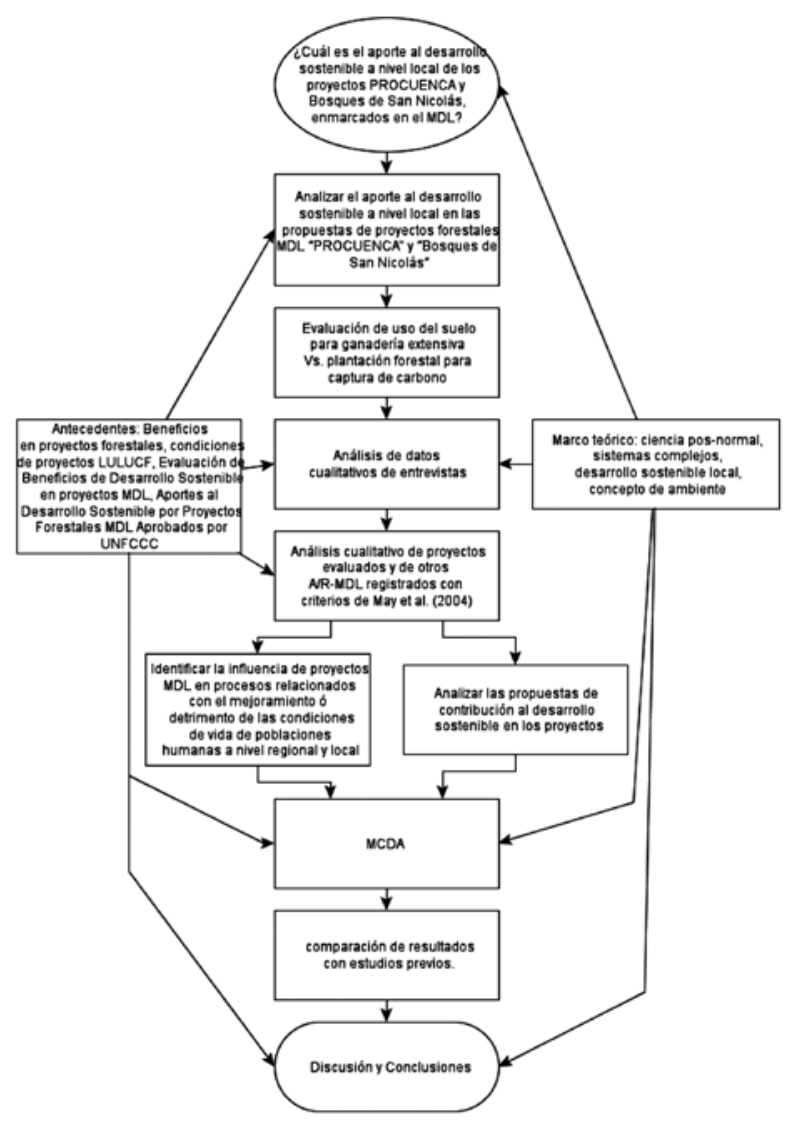

Ilustración 1. Diagrama de flujo de la metodología utilizada $^{1}$

Se asumió que el desarrollo sostenible es un tema donde se encuentran posturas e intereses legítimos y contrastantes, donde los argumentos que aporte la ciencia son tan sólo una postura más en medio de un escenario complejo (De Montis, De Toro, Droste, Omann \& Stagl, 2000).
Los actores en torno a un problema de estudio de este tipo no pueden ser concebidos en forma homogénea dado que no poseen la misma capacidad de influencia en la toma de decisiones y no tienen acceso a la misma información. Las observaciones realizadas dependerán en gran medida de los actores que se elijan para ser incluidos en el estudio. En consecuencia, no se trata de una población cuya capacidad de decisión y la participación de uno u otro actor se comporten completamente al azar. Los grupos de interés se pueden concebir como sistemas complejos organizados en los que la estructura jerárquica influye en la toma de decisiones, y en el acceso y manejo de la información. En consecuencia, la muestra de actores realizada se enfocó en líderes y funcionarios de cargos decisorios que estaban en capacidad de brindar panoramas integrales y contextualizados de su grupo de interés y el problema de estudio. Estas consideraciones permiten que el tamaño de muestra sea menor en comparación a diseños experimentales que asumen aleatoriedad en la participación de los actores en la toma de decisiones y homogeneidad de los mismos dentro de los grupos de interés, contrario a la postura de informantes clave.

En los estudios de caso analizados se contactaron informantes clave, de los distintos grupos de interés relevantes en el diseño e implementación de los proyectos; para los dos proyectos se identificaron como grupos de interés: los desarrolladores del proyecto, las instituciones de orden local y los habitantes vinculados a las actividades de captura de carbono. Los informantes elegidos fueron líderes y funcionarios que representaban la opinión de un sector ó grupo de interés de cada uno de los proyectos; por lo tanto, el criterio de selección no fue de tipo estadístico sino que se parte de los intereses y la capacidad de influencia y negociación que cada uno de los sectores posee y que se ve representado en los líderes de cada grupo de interés. Este criterio parte de las consideraciones del marco epistemológico de la ciencia post-normal, ya que se reconoce en cada grupo de interés una postura legítima que este busca defender.

1 Fuente: elaborada por los autores. 
A partir de estudios previos se definió un conjunto inicial de criterios. Entre los modelos internacionales de evaluación, se utilizan criterios de: South South North (The South South North, 1999), The Gold Standard (The Gold Standard Foundation, 2009; The Gold Standard Foundation, 2008), la Evaluación Multi-Atributo de MDL (Sutter, 2003) y la propuesta taxonómica para Evaluación de Beneficios de Olsen \& Fenhann (2008). Al compararlos se encuentra que estos métodos de evaluación comparten en total diez criterios que se utilizan en la evaluación.

Utilizando la entrevista semi-estructurada y abierta, a los informantes clave se les preguntó acerca del desempeño de los proyectos de acuerdo a los criterios que fueron definidos. Adicionalmente, se realizaron visitas a las áreas de implementación de los proyectos, identificando problemas y ventajas de carácter técnico y operativo de la ejecución de los mismos. Esta evaluación se complementó con discusiones realizadas con los entrevistados en torno a otros aspectos relevantes respecto al aporte al desarrollo sostenible local y el éxito de los proyectos.

Luego de colectada la información generada a partir de las respuestas de los entrevistados en relación con los criterios y la evaluación de los proyectos que realizaron, estos datos se gestionaron siguiendo a Kenyon (2007) y Prato (2003) con el fin de realizar una evaluación general de los proyectos, aplicando los métodos de suma de rangos de peso (RS) y la ordenación de rangos por centroide (ROC).

\section{Análisis de dos proyectos forestales MDL en la región andina en Colombia}

\subsection{Proyecto MDL Cuenca del río Chinchiná PROCUENCA}

El proyecto está ubicado al sur del departamento de Caldas entre 800 metros y 3000 metros de altitud sobe el costado occidental de la cordillera central, abarca cinco municipios y representa un ecosistema estratégico que abastece de bienes y servicios ambientales a la subregión que produce el 70\% del PIB del Departamento de Caldas y es fuente de una rica y variada biodiversidad. El proyecto es administrado financieramente por Infimanizales, abarca 4538 hectáreas para 2 millones 181 mil 676 certificados tCER; ha celebrado 380 contratos con propietarios de terrenos, recibiendo fondos de instituciones locales por más de 25 mil millones de pesos en 6 años y recientemente contó con ayuda internacional del programa MIDAS de USAID para capacitación. En agosto de este año fue galardonado por su aporte a la responsabilidad social y actualmente se encuentra en trámite de aprobación ante Naciones Unidas.

\subsection{Proyecto MDL Valle de San Nicolás}

Este proyecto se denomina Modelo de Financiación Alternativo para el Manejo Sostenible de los Bosques de San Nicolás. Se ubica en la Región Valles de San Nicolás, en el suroriente de Antioquia entre 1800 metros y 2700 metros de altitud, también sobre el flanco occidental de la cordillera central y abarca el altiplano antioqueño denominado batolito antioqueño; comprende 9 municipios que conforman la zona con mayor desarrollo económico e industrial del departamento después de Medellín y del valle de Aburrá.

El proyecto es administrado por la corporación Masbosques y surge de un contrato de compraventa con el banco mundial, con un área inicial pactada de 1410 ha por un millón de dólares para 120 mil tCER y tiene un total proyectado de 442 mil toneladas de $\mathrm{CO} 2$ en 21 años. El proyecto lleva cinco años en fase de diseño y cerca de un año de implementación, actualmente está en fase de formulación del documento de propuesta para Naciones Unidas.

\subsection{Aplicación de criterios}

Aplicando la metodología mencionada, a cada entrevistado se le pidió que calificara el proyecto de acuerdo a la contribución que éste hacía a los criterios previamente establecidos y comentados. La manera en que se calificaron estas alternativas, fue asignando un número de uno a diez, siendo uno perjuicios muy altos y diez aportes excelentes y directos frente al criterio evaluado. No se presentaron casos en que el entrevistado se negara a calificar el aporte a un criterio, ni casos en que no sabía cuál podía ser el efecto de la alternativa evaluada. 
Para la asignación de puntajes se pidió a los entrevistados que iniciaran la actividad calificando la alternativa de ganadería extensiva, actividad productiva tradicional, y con base en esta, que calificaran los beneficios que esperarían al implementar el proyecto forestal hasta 2012, año en que finaliza el período de compromiso del proyecto. Adicionalmente, los comentarios que los entrevistados realizaron durante la calificación de los criterios también fueron recogidos y analizados como datos cualitativos del estudio.
La Tabla 1 muestra los resultados de la calificación de las alternativas evaluadas: el uso del predio con el proyecto (plantación forestal) y el uso sin proyecto (ganadería extensiva). A todos los entrevistados se les dio el mismo peso a la hora de realizar los cálculos. Los puntajes que se presentan en la primera parte son los valores de las medias de los puntajes signados y en segundo lugar, las medianas de los mismos puntajes.

Tabla 1. Medias y medianas de puntajes para cada alternativa (con y sin proyecto) por cada criterio²

\begin{tabular}{|c|c|c|c|c|}
\hline \multirow[b]{2}{*}{ Media de puntajes } & \multicolumn{2}{|c|}{ Procuenca } & \multicolumn{2}{|c|}{ Masbosques } \\
\hline & Con proyecto & Sin proyecto & Con proyecto & Sin proyecto \\
\hline \multicolumn{5}{|l|}{ Ambiente } \\
\hline Aire & 8,50 & 3,50 & 7,44 & 4,00 \\
\hline Agua & 7,67 & 4,06 & 6,33 & 5,00 \\
\hline Suelo & 7,86 & 5,00 & 8,00 & 4,67 \\
\hline Biodiversidad & 7,83 & 4,33 & 8,11 & 4,33 \\
\hline \multicolumn{5}{|l|}{ Económico } \\
\hline Inversión & 5,67 & 5,00 & 5,33 & 6,00 \\
\hline Empleo & 5,00 & 2,50 & 4,67 & 2,67 \\
\hline Riqueza & 7,17 & 4,83 & 8,00 & 5,67 \\
\hline Tecnología & 5,00 & 4,72 & 4,00 & 4,00 \\
\hline \multicolumn{5}{|l|}{ Social } \\
\hline Fuentes de ingreso & 7,17 & 2,83 & 7,00 & 2,67 \\
\hline Mejores empleos & 4,67 & 3,50 & 4,33 & 3,33 \\
\hline \multicolumn{5}{|l|}{ Ambiente } \\
\hline Aire & 8,50 & 3,50 & 7,00 & 4,00 \\
\hline Agua & 7,50 & 4,00 & 6,00 & 5,00 \\
\hline Suelo & 7,50 & 5,00 & 8,00 & 5,00 \\
\hline Biodiversidad & 8,00 & 4,00 & 9,00 & 5,00 \\
\hline \multicolumn{5}{|l|}{ Económico } \\
\hline Inversión & 6,00 & 4,50 & 5,00 & 6,00 \\
\hline Empleo & 5,00 & 2,50 & 5,00 & 3,00 \\
\hline Riqueza & 7,50 & 5,00 & 8,00 & 6,00 \\
\hline Tecnología & 5,00 & 5,00 & 4,00 & 4,00 \\
\hline \multicolumn{5}{|l|}{ Social } \\
\hline Fuentes de ingreso & 7,00 & 3,00 & 7,00 & 3,00 \\
\hline Mejores empleos & 4,50 & 3,50 & 4,00 & 3,00 \\
\hline
\end{tabular}

2 Elaborada por los autores a partir de las respuestas aportadas por los entrevistados. 
Para la interpretación de los resultados obtenidos a partir de los datos cuantitativos es importante tener en cuenta la racionalidad en intereses que se expresan en los datos y es en esta medida que resulta importante el análisis de la información cualitativa que se colectó como se muestra a continuación.

\subsection{Asignación de pesos a criterios}

Para el análisis de los datos colectados se incluyeron los pesos que los entrevistados asignaron a los criterios utilizados en la evaluación de los proyectos. La manera en que los entrevistados llevaron a cabo la asignación de pesos fue a través de la ordenación del más importante al menos importante de los criterios por medio de tarjetas didácticas, una para cada criterio. Al final de la ordenación de criterios también se comentó el orden asignado y se recogieron las razones y observaciones expresadas por los entrevistados.

La Tabla 2 presenta los pesos de los criterios calculados para el total de entrevistados de cada proyecto. Para la asignación de peso a los criterios se siguieron las recomendaciones de Kenyon (2007) y Jia, Fischer \& Dyer (1998); aplicando los métodos de suma de rangos de peso (RS) y la ordenación de rangos por centroide (ROC). El primero requiere información acerca de la ordenación de los criterios, realizada por los entrevistados; donde a cada criterio se le asigna un peso en proporción a su posición. En la segunda opción, se asume que si no se tiene más información que la calificación de alternativas realizada por los entrevistados, los pesos se distribuyen uniformemente sobre un simplex de pesos de ordenación de rango (Kenyon, 2007). Las ecuaciones (1) y (2) corresponden respectivamente al cálculo de RS y ROC.

$w_{i}=\frac{\text { rango inverso }}{\text { suma de rangos }}=\frac{2(m+1-j)}{m(m+1)}$

$w_{i}=\frac{1}{m} \sum_{j=i}^{m}(1 / j)$

$w$ Peso

$m$ Número de criterios

j Posición de criterios

3 Elaborada por los autores.
Tabla 2. Ponderación de criterios usando los métodos de suma de rangos (RS) y centroide por ordenación de rangos $(\mathrm{ROC})^{3}$

\begin{tabular}{|l|l|c|c|c|c|}
\hline \multirow{2}{*}{ Criterio } & \multicolumn{2}{c|}{ Procuenca } & \multicolumn{2}{c|}{ Masbosques } \\
\cline { 2 - 6 } & RS & ROC & RS & ROC \\
\hline \multicolumn{2}{|c|}{ Ambiente } & & & & \\
\hline \multicolumn{2}{|l|}{ Aire } & 0,04 & 0,23 & 0,03 & 0,20 \\
\hline & Agua & 0,14 & 0,20 & 0,09 & 0,19 \\
\hline & Suelo & 0,14 & 0,17 & 0,12 & 0,18 \\
\hline \multicolumn{2}{|l|}{ Biodiversidad } & 0,08 & 0,20 & 0,13 & 0,23 \\
\hline \multicolumn{2}{|l|}{ Económico } & & & & \\
\hline \multicolumn{2}{|l|}{ Inversión } & 0,12 & 0,20 & 0,13 & 0,19 \\
\hline & Empleo & 0,08 & 0,36 & 0,13 & 0,30 \\
\hline & Riqueza & 0,14 & 0,22 & 0,14 & 0,16 \\
\hline & Tecnología & 0,06 & 0,22 & 0,06 & 0,28 \\
\hline \multicolumn{2}{|l|}{ Social } & & & & \\
\hline & Fuentes de ingreso & 0,14 & 0,28 & 0,13 & 0,26 \\
\hline & Mejores empleos & 0,05 & 0,31 & 0,06 & 0,29 \\
\hline
\end{tabular}

El cálculo de RS se utiliza la Ecuación (1), para hallar la puntuación del rango inverso de cada criterio para cada entrevistado, se suman y se dividen sobre el total de entrevistados. Para el cálculo de ROC se utiliza la Ecuación (2), para hallar el peso de cada criterio de acuerdo a la puntuación de alternativas de cada entrevistado, se suman y se dividen sobre el total de entrevistados (Kenyon, 2007). De esta manera se le asignan pesos a los datos en bruto, obteniendo dos valores que fueron utilizados para comparar los resultados obtenidos para cada criterio.

Para la elección de la forma de ponderación más apropiada se realizó análisis de regresión lineal con los resultados obtenidos de aplicar los métodos utilizados (Ver Tabla 2), lo que se complementó con evidencias de estudios previos que definen la mejor manera para llevar a cabo la ponderación de los criterios utilizados en un MCDA. A partir de los dos métodos recomendados en estudios previos, se analizan tres posibilidades: i) utilizar los dos métodos y 
comparar resultados, ii) la elección de RS ó iii) la elección de ROC. Para demostrar cuál de estas tres es la más apropiada se realizaron análisis de correlación lineal con el fin de determinar cuál de las opciones muestra mayor relación entre los resultados generados por las entrevistas.

La ilustración 2 muestra que existen correlaciones demasiado bajas entre resultados de RS y ROC en ambos estudios de caso (valores de R2 de $0.17 \mathrm{y}$
0.03 para PROCUENCA y Bosques de San Nicolás respectivamente), lo mismo ocurre al analizar la correlación que existe entre resultados de los estudios de caso con ROC (valor de R2 de 0.04), como lo muestra la Ilustración 3. Por el contrario la correlación de resultados entre los estudios de caso con RS es apreciable (valor de R2 de 0.56). Esto demuestra que la opción de ponderación de criterios más apropiada para esta investigación es el método RS.

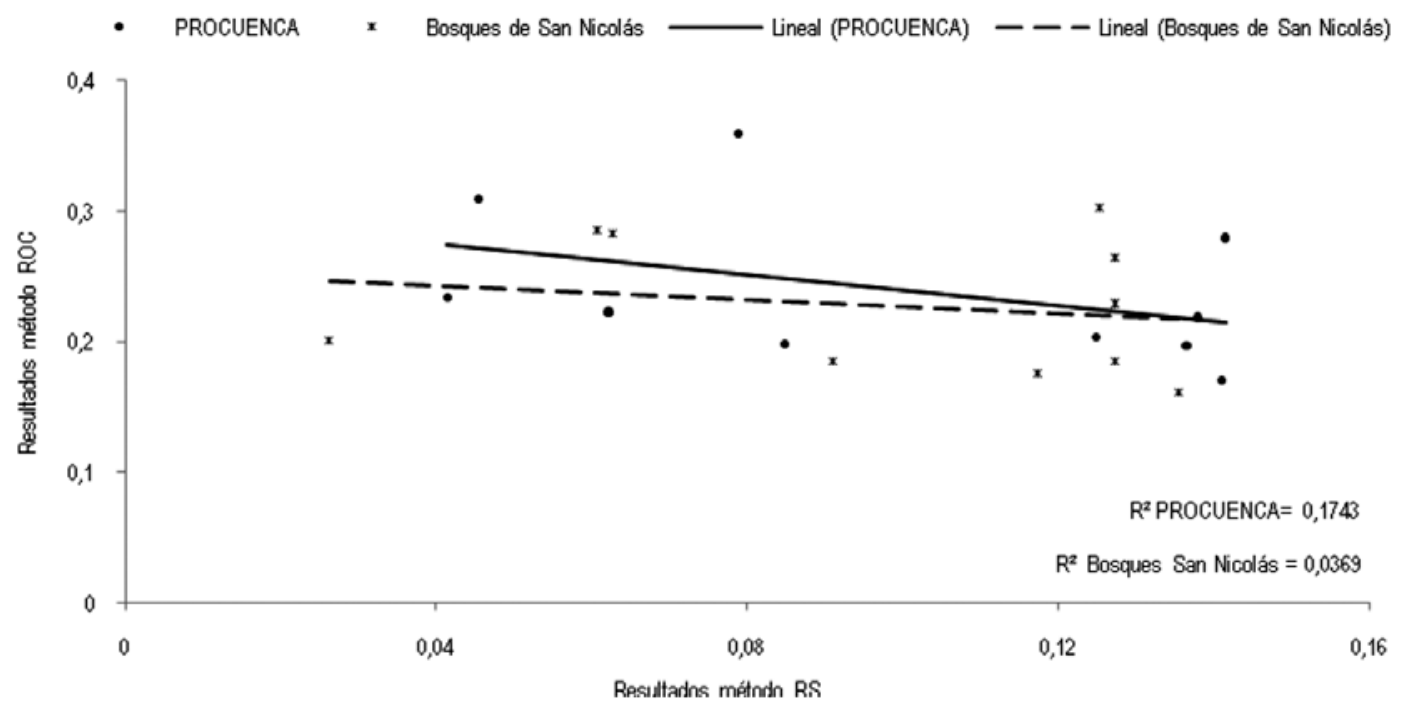

Ilustración 2. Relaciones entre resultados de métodos de tratamiento de criterios utilizados en el MCDA (RS y ROC) ${ }^{4}$

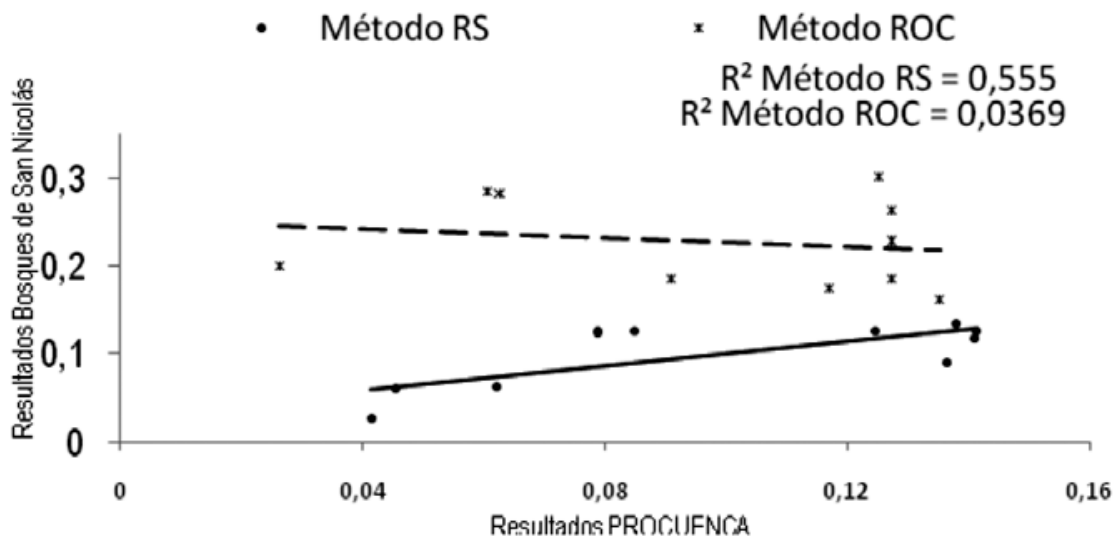

Ilustración 3. Relaciones entre resultados hallados en los estudios de caso con el método RS y ROC para el tratamiento de criterios ${ }^{5}$

Elaborada por los autores.

Elaborada por los autores. 
Al comparar los resultados de RS con los que arroja el método ROC, se aprecia en primer lugar que los pesos que arroja ROC en general son mayores en comparación con los que genera RS y en segundo lugar que los resultados son distintos al comparar los pesos asignados en cada método.

En el artículo se tomará como valor principal el método RS, ya que el análisis del aporte al desarrollo sostenible que desarrolla el presente estudio, busca considerar el mayor número de criterios en las distintas dimensiones examinadas (ambiental, social y económica) Jia et al. (1998). También se asume que hay diversos factores que intervienen en la sostenibilidad local, por lo que es preferible incorporar en el análisis un mayor número de criterios. Los resultados generados por $\mathrm{ROC}$ se tienen en cuenta para analizar las diferencias entre puntuaciones de las alternativas y la interpretación de la información cualitativa.

\subsection{Combinación de pesos y puntajes}

Para el cálculo del puntaje agregado para cada alternativa y en cada caso estudiado, se utilizó una función lineal aditiva que simplifica el análisis, la escogencia de este método y su aplicación se basó en los trabajos de Prato (2003) y Kenyon (2007). La Ecuación (3) muestra la manera en que se estandarizaron los puntajes por criterio para cada alternativa y la Ecuación (4) presenta la combinación de pesos y puntajes realizada; esta última parte considera que la utilidad que aporta un criterio adicional es independiente de la cantidad de criterios (Prato, 2003). Los resultados son presentados en la Tabla 3 y calculados multiplicando la media o la mediana, según el caso, del puntaje calculado y dividido entre todos los participantes por el peso total de todos los participantes.

$S_{k i}=\frac{x_{k i}-\min _{i} x}{\max _{i} x_{k i}-\min _{i} x_{k i}}$

Para atributos positivos donde más es mejor

$u_{k}=\sum_{i=1} w_{i} s_{k i}(k=1, \ldots, n)$ $x_{k i} \quad$ Valor bruto del i-écimo criterio para la k-écima opción

$s_{k i} \quad$ Valor estandarizado del i-écimo criterio para la k-écima opción

$u_{k} \quad$ Valor de utilidad para la k-écima opción

$m$ Número de criterios

$w_{i}$ Peso del i-écimo criterio

$i \quad$ Un criterio específico

$k$ Una opción específica

Los resultados del análisis multicriterio muestran que la implementación de plantaciones forestales en las áreas potenciales arroja cerca del doble de beneficios: medias de 0,67 versus 0,32 en el proyecto PROCUENCA y de 0,63 versus 0,38 en el proyecto de Bosques de San Nicolás, en comparación con la utilización de estos mismos terrenos para ganadería extensiva hasta el final del período de compromiso; lo que se confirma por los valores de media y mediana similares, soportando de esta manera dicha conclusión.

\section{Información cualitativa colectada}

La Tabla 4 sintetiza los beneficios que aporta cada proyecto en la escala local de acuerdo a la información suministrada por los entrevistados; esta tabla fue construida de acuerdo con los abordajes metodológicos de Grieg-Gran (2005) y May et al. (2004). Al realizar la comparación se identifican los beneficios que impactan en la escala local y que están en concordancia con los planteamientos de Carrizosa (2005). Los beneficios aquí evaluados involucran un componente ambiental, social, y económico.

En términos generales, los proyectos fomentaron un mejor manejo de plantaciones forestales y mayores oportunidades de mercado y en consecuencia bosques con mayor valor; relacionado con esto, se generarán beneficios en el aumento de la fertilidad del suelo, reducción de procesos de erosión, aumento de la disponibilidad de agua en las cuencas y promoción de una cultura forestal. 
Tabla 4. Evaluación cualitativa de los proyectos forestales MDL bajo estudio. Si presenta el atributo: 1 , si no: $0^{6}$

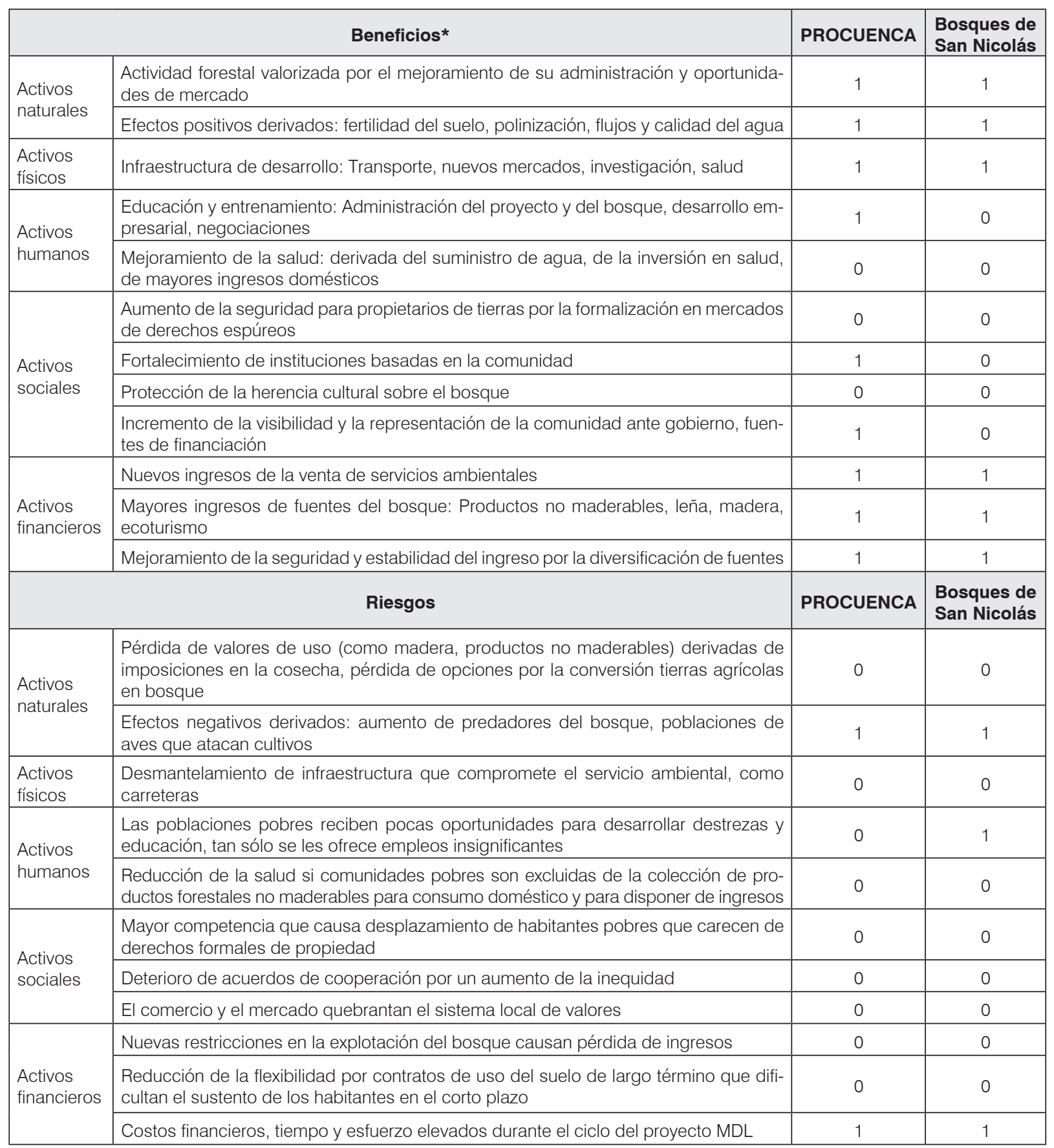

6 Elaborada por los autores. 
En el aporte social se encontraron diferencias de concepción entre los grupos de interés. En el caso del proyecto PROCUENCA todos los grupos de interés concuerdan en que habrá un aporte en educación y entrenamiento, en el fortalecimiento de organizaciones sociales, en la visibilidad de la comunidad frente a instituciones estatales y en acceso a fuentes de financiación. Por otro lado, en el proyecto de Bosques de San Nicolás, los campesinos entrevistados no reconocen que existan aportes significativos de tipo social.

Respecto al aspecto económico, la implementación de los proyectos generará infraestructura para el desarrollo, ampliación de mercados, promoción de la oferta de servicios ambientales y generación de conocimiento necesario para el mantenimiento y éxito de las plantaciones forestales.

Resultado de lo anterior, la generación de nuevas fuentes de ingreso por la venta de servicios ambientales, aumentó del atractivo turístico y del paisaje, y reducción de costos por el acceso a madera, leña y otros servicios ambientales. En definitiva, los grupos de interés reconocen una mayor estabilidad económica de las comunidades y consideran las plantaciones forestales como un renglón a considerar en la economía rural, hecho que antes no se identificaba.

Los entrevistados de todos los grupos de interés de ambos proyectos, reconocieron riesgos por: i) costos financieros, tiempo y esfuerzo elevados a lo largo del ciclo del proyecto MDL, ii) aumento de poblaciones de animales predadores del bosque $y$ de animales que pueden atacar o deteriorar cultivos. Adicionalmente, en el proyecto de Bosques de San Nicolás los campesinos expresaron preocupación porque no se identifican oportunidades claras para el desarrollo de destrezas y educación y creen que este tipo de beneficios puede limitarse a la oferta de empleos temporales al interior del proyecto. Respecto a los riesgos financieros por los costos elevados que se pueden generar, en entrevistas con líderes y representantes de los grupos de interés se pudo evidenciar la necesidad de que los proyectos MDL sean diseñados como una extensión adicional a proyectos con metas que tienen financiación asegurada, donde las metas de la captura de carbono se puedan generar de manera conexa. De esta manera se concluye de lo expresado, que la generación de beneficios por la captura de carbono en sí puede no ser la meta de un proyecto forestal que busque mantenerse en el largo plazo, ya que las características del mercado de carbono y de la demanda de servicios ambientales suponen alta incertidumbre.

\section{Discusión}

El análisis de los datos y experiencias de los proyectos analizados aportan evidencias que fortalecen el enfoque del desarrollo sostenible local, gracias a las interrelaciones presentes entre los grupos de interés y la organización: local, nacional e internacional, en las dimensiones: ambientales, sociales y económicas.

A nivel local se ha encontrado que el desarrollo de los proyectos analizados ha generado conocimiento y capacidades valiosas en la implementación y manejo de bosques y suelos, que han hecho posible el surgimiento de instituciones y organizaciones locales con la potencialidad de involucrarse en mercados de bienes y servicios ambientales.

Por otra parte, se identificó un conflicto de intereses entre la escala global que busca la reducción de emisiones costo-eficiente de GEI y la escala local, en donde los intereses se enfocan en el aporte al desarrollo sostenible. En este sentido, se considera necesario garantizar institucionalmente que los proyectos MDL contribuyan a los objetivos definidos, particularmente en lo relacionado con el desarrollo sostenible local en los países receptores de tales proyectos.

Adicionalmente, los proyectos MDL pueden aumentar sus beneficios locales en las zonas estudiadas si superan las siguientes barreras: carencia de conocimientos y recursos técnicos suficientes para afrontar numerosos obstáculos operativos tales como la ausencia de semillas y plántulas de especies nativas que garanticen éxitos similares a las variedades comerciales de especies foráneas; escaso conocimiento sobre control de plagas en las plantaciones; elevados costos de implementación de los proyectos 
cuando se incluyen especies nativas, y altos costos de transacción en los trámites de registro y aprobación ante la Junta Ejecutiva en MDL de la Convención Marco de las Naciones Unidas sobre Cambio Climático (UNFCCC por sus siglas en inglés).

Se evidenció que las instituciones son importantes en el establecimiento y promoción de procesos locales, pero que el mantenimiento de estos se da por parte de organizaciones civiles y comunitarias que se apropian de los procesos; lo que se manifiesta en que en los dos proyectos analizados se integra la participación como elemento fundamental. En PROCUENCA, el fortalecimiento de la participación local es uno de los objetivos del proyecto, en el caso de Bosques de San Nicolás, la organización desarrolladora del proyecto, está conformada en su junta directiva por representantes de diferentes sectores y entre ellos por las juntas de acción comunal de los municipios donde se ha previsto las zonas de establecimiento de plantaciones.

Partiendo de la ausencia de estándares internacionales en desarrollo sostenible en el marco del MDL, el desarrollo sostenible local es un enfoque que enriquece la discusión y aporta argumentos para mejorar el diseño de propuestas de proyectos forestales MDL con el objetivo de impactar positivamente las comunidades locales. Permite además, la utilización de criterios de valoración local, lo que enriquece el análisis multicriterio incorporando elementos de tipo político y participativo e intereses y preferencias de grupos de interés, en el diseño y evaluación de estos proyectos.

Para el diseño de proyectos forestales MDL que aporten al desarrollo sostenible local, es importante que estos se enmarquen en una política de promoción forestal con mecanismos de gestión e incentivos, teniendo en cuenta las condiciones nacionales y el contexto local para cumplir exitosamente con el doble objetivo que persigue el protocolo de Kioto a través del MDL, potenciando así la contribución al desarrollo sostenible local en las naciones hospederas de este tipo de proyectos.

Teniendo en cuenta los beneficios y barreras asociados a estos proyectos, el diseño participativo de programas forestales MDL puede contribuir a la sostenibilidad local por los procesos de cofinanciación y desarrollo de conocimiento. Estos procesos se pueden fortalecer en el contexto nacional dada la coexistencia de un conjunto de condiciones propias de un país intertropical con vocación forestal, alta diversidad de climas y especies, lo que sumado a la riqueza organizativa y cultural puede sustituir la utilización depredadora actual del suelo por procesos de desarrollo sostenible local.

La implementación de los proyectos forestales evaluados ha influido positivamente en varios procesos que mejoran condiciones de vida local y regional, estos son presentados en la Tabla 4 . El hecho mismo de afrontar este tipo de proyectos mostró las potencialidades con que se cuenta a nivel regional y nacional. A nivel local se ha encontrado que el desarrollo de los proyectos analizados ha generado conocimiento, capacidades valiosas en la implementación, manejo de bosques, suelos, e hizo posible el surgimiento de instituciones y organizaciones locales con la potencialidad de desarrollar mercados de bienes y servicios ambientales.

\section{Conclusiones}

El presente estudio permite concluir que en el diseño de los proyectos evaluados se involucraron múltiples actores locales, lo cual fortaleció institucionalmente los proyectos e incrementó su compromiso en el desarrollo; no obstante, una mayor participación implicó a su vez, la existencia de conflictos de interés. Dada esta situación, el MCDA demostró ser un abordaje adecuado, ya que permitió una evaluación que incorporó posturas e intereses contrarios.

Por otro lado, mientras que en el diseño del proyecto se dio una clara valoración al mejoramiento de suelos, generación de riqueza en áreas deprimidas y generación de nuevas fuentes de ingreso; se encontró que los procesos claramente fomentados en el desarrollo de estos proyectos fueron dos: desarrollo del potencial forestal y generación de conocimiento. Como resultado los proyectos muestran un aporte al mejoramiento de las condiciones locales. 
Aunque los aportes identificados son significativos en relación con el desarrollo sostenible local gracias a la vinculación de instituciones y organizaciones civiles del ámbito local que fueron determinantes en el establecimiento y ejecución de los proyectos, se identificaron necesidades técnicas, de participación y organización local que requieren atención durante la ejecución de estos proyectos. Del mismo modo, como evidenció Graichen (2005), si el precio de los certificados CER es bajo, los menores ingresos económicos por tCER generan un conflicto con las oportunidades para aportar beneficios adicionales en áreas rurales en el diseño de estos proyectos. Por eso el MDL debe garantizar un precio justo para los desarrolladores y propietarios de los proyectos que permita a éste convertirse en un mecanismo que plantee una alternativa viable de cofinanciación de proyectos forestales.

En consecuencia, los proyectos forestales MDL evaluados han generado una contribución al desarrollo sostenible local en áreas rurales dado que impactaron el paisaje por un mejor uso y manejo del territorio; han promovido la generación de nueva infraestructura que permite acceder a mercados de bienes y servicios ambientales y diversificar la producción e incentivaron la participación de los titulares del suelo, frente a la alternativa de manejo del suelo no sostenible que tradicionalmente se realiza en la región.

Se recomienda diseñar proyectos forestales MDL enmarcados en programas forestales que cuenten con fuentes de financiación diversas, para que puedan cumplir con metas sociales y de desarrollo sostenible ambiciosas, teniendo en cuenta las condiciones nacionales y el contexto rural para así lograr aportes al desarrollo sostenible local y cumplir exitosamente con el doble objetivo que persigue el Protocolo de Kioto a través del MDL y superar las limitaciones que impone la competencia basada en el carácter costo efectivo que domina a nivel internacional en la utilización de este instrumento.

De los resultados presentados en el artículo, se generan las siguientes preguntas que tienen como propósito alentar la investigación en el tema: ¿Cómo evaluar la importancia de las poblaciones locales en el mantenimiento de proyectos de largo plazo?
¿Bajo qué condiciones se pueden aprovechar escenarios de crisis económica en territorios rurales para promover iniciativas de uso del suelo ambientalmente más sostenibles aunque menos rentables en términos financieros? ¿La interacción entre instituciones internacionales y locales facilita el establecimiento de iniciativas de largo plazo?

\section{Referencias}

Borja, J. \& Castells, M. (1998). Local y global. Barcelona: Taurus. Brown, K., Adger, W. N., Boyd, E., Corbera, E., \& Shackley, S. (2004). How do CDM projects contribute to sustainable development? (Rep. No. 16). Norwich, United Kingdom: Tyndall Centre for Climate Change Research.

Carmona-Maya, S. I. (2001). Modelos culturales y sostenibilidad. In Modelos culturales y sostenibilidad Medellín, Colombia: Instituto de Estudios Ambientales IDEA, Universidad Nacional de Colombia sede Medellín.

Carrizosa, J. (2000). ¿Qué es el ambientalismo? La visión ambiental compleja. Bogotá D.C.: Giro Editores Ltda.

Carrizosa, J. (2001). Colombia, de lo imaginario a lo complejo; reflexiones y notas acerca de ambiente, desarrollo y paz. Bogotá D.C.: Panamericana Formas e Impresiones S.A.

Carrizosa, J. (2005). Desequilibrios territoriales y sostenibilidad Local. Bogotá D.C.: Universidad Nacional de Colombia. Instituto de Estudios Ambientales (IDEA).

Clark, J. G. (1995). "Economic development vs. Sustainable societies: reflections on the players in a crucial contest". En: Annual Review of Ecology and Systematics, 26: 225-248.

De Montis, A., De Toro, P., Droste, B., Omann, I., \& Stagl, S. (2000). Criteria for quality assessment of MCDA methods". En: Transitions Towards a Sustainable Europe (pp. 1-30). Vienna, Austria: Universitat Autonoma Barcelona, European Commission Environment and Climate Programme.

Echeverri, G., Naranjo, B., \& Uribe, D. (2003). Las herramientas multicriterio para la evaluación de proyectos productivos sostenibles en los bosques de San Nicolás (ANT.). Tesis de grado no publicada. Medellín, Colombia: Universidad Nacional de Colombia sede Medellín, Facultad de Ciencias Humanas y Económicas.

Escobar, A. (1996). Encountering development; the making and unmaking of the third world. New Jersey: Princeton University Press.

Fan, T., Liu, D. \& Tzeng, G. (2007). Decision Support Rough setbased logics for multicriteria decision analysis. En: European Journal of Operational Research, 182: 340-355.

Grieg-Gran, M., Porras, I., \& Wunder, S. (2005). How Can Market Mechanisms for Forest Environmental Services Help the Poor? Preliminary Lessons from Latin America. En: World Development, 33: 1511-1527.

Guimarâes, R. (2001). Fundamentos territoriales y biorregionales de la planificación. Santiago: Comisión Económica para América Latina y el Caribe. Medio Ambiente y Desarrollo, 39.

Hepburn, C. (2007). Carbon Trading: A Review of the Kyoto Mechanisms. En: The Annual Review of Environment and Resources, 32: 375-393. 
Jamal, F. (2006). Sustainability assessment in the Clean Development Mechanism (CDM): Current state and opportunieties for improvement. Tesis de grado no publicada. Vancouver, BC Canada: The University of British Columbia, The Faculty of Graduate Studies, Resource Management and Environmental Studies, Master of Science.

Jia, J., Fischer, G., \& Dyer, J. (1998). Attribute weighting methods and decision quality in the presence of response error: a simulation study. En: Journal of Behavioral DecisionMaking, 11: 85-105.

Keeler, A. (2008). Industrialized-Country Mitigation Policy and Resource Transfers to Developing Countries: Improving and Expanding Greenhouse Gas Offsets. Cambridge, Mass., USA: Harvard Kennedy School.

Kenyon, W. (2007). Evaluating flood risk management options in Scotland: A participant-led multi-criteria approach. En: Ecological Economics, 64: 70-81.

Landell-Mills, N. \& Porras, I. (2002). Silver bullet or fools' gold? A global review of markets for forest environmental services and their impact on the poor. London, UK: International Institute for Environment and Development.

Martinez-Alier, J., Munda, G., \& O'Neill, J. (1998). Weak comparability of values as a foundation for ecological economics. En: Ecological Economics, 26: 277-286.

May, P.H. Boyd, E. Veiga, F. \& Chang, M. (2004). Local sustainable development effects of forest carbon projects in Brazil and Bolivia. A view from the field. International Institute for Environment and Development, London.

Moncayo, E. (2001). Evolución de los paradigmas y modelos interpretativos del desarrollo territorial. Santiago de Chile: Comisión Económica para América Latina y el Caribe CEPAL-IIpes.

Munda, G. (1993). Multiple-Criteria Decision Aid: Some Epistemological Considerations. En: Journal of Multi-Criteria Decision Analysis, 2: 41-55.

Munda, G. (2004a). Métodos y procesos multicriterio para la evaluación social de las políticas públicas. En: Revista de la Red Iberoamericana de Economía Ecológica, 1: 31-45

Munda, G. (2004b). Social multi-criteria evaluation: Methodological foundations and operational consequences. En: European Journal of Operational Research, 158: 662-677.

Munda, G. (2009). A conflict analysis approach for illuminating distributional issues in sustainability policy. En: European Journal of Operational Research, 194: 307-322.

Olsen, K. H. \& Fenhann, J. (2008a). A Reformed CDM. Roskilde, Denmark: The Technical University of Denmark, UNEP Ris $\varnothing$ Centre, Risø National Laboratory for Sustainable Energy.

Olsen, K. H. \& Fenhann, J. (2008b). Sustainable development benefits of clean development mechanism projects A new methodology for sustainability assessment based on text analysis of the project design documents submitted for validation. En: Energy Policy, 36: 2819-2830.
Prato, T. (2003). Multiple-attribute evaluation of ecosystem management for the Missouri River system. En: Ecological Economics, 45: 297-309.

Sabogal, J. (2008). Aproximación y cuestionamientos al concepto Responsabilidad Social Empresarial. En: Revista Facultad de Ciencias Económicas: Investigación y Reflexión, XVI (1): 179-195.

Sabogal, J. \& Hurtado, E. (2009). La historia se repite: una visión del desarrollo y del desarrollo sostenible. En: Revista Facultad de Ciencias Económicas: Investigación y Reflexión, XVII (2): 195-216.

Sagar, A. D. (2000). Capacity development for the environment: a view for the south, a view for the north. En: Annual Review of Environment Resources, 25: 377-439.

Stern, N. (2007). El informe Stern: la verdad del cambio climático. Barcelona, España: Paidós.

Sutter, C. (2003). Sustainability Check-Up for CDM Projects. Berlin, Germany: Wissenschaftlicher Verlag.

Sutter, C. \& Parreño, J. C. (2007). Does the current Clean Development Mechanism (CDM) deliver its sustainable development claim? An analysis of officially registered CDM projects. En: Climatic Change, 84: 75-90

Teixeira, M., Murray, M., \& Carvalho, M. (2006). Assessment of land use and land use change and forestry (LULUCF) as CDM projects in Brazil. En: Ecological economics, 60: 260-270.

The Gold Standard Foundation (2008). Annexes to toolkit. The Gold Standard Foundation. Recuperado el 18-01-2010, Disponible en: http://www.cdmgoldstandard.org/

The Gold Standard Foundation (2009). "Gold Standard Toolkit". The Gold Standard Foundation. Recuperado el 18-01-2010, Disponible en: http://www.cdmgoldstandard.org/

The South South North. (1999). Sustainable Development Appraisal \& Ranking Matrix Tool. Cape Town, South Africa: South South Noth.

United Nations Framework Convention on Climate Change. (1997). Kyoto Protocol. Recuperado el 18-01-2010, Disponible en: http://unfccc.int.

United Nations Framework Convention on Climate Change (2002). Report of the Conference of the Parties on its Seventh Session, Held at Marrakesh from 29 October to 10 November 2001. (Rep. No. FCCC/CP/2001/13). Marrakesh, Morocco: United Nations. Recuperado el 18-01-2010, Disponible en: http://unfccc.int.

United Nations Framework Convention on Climate Change (2004). Report of the Conference of the Parties on its Ninth Session, Held at Milan from 1 to 12 December 2003 (Rep. No. FCCC/CP/2003/6). Milan, Italy: United Nations Framework Convention on Climate Change. Recuperado el 18-012010, Disponible en: http://unfccc.int.

Van der Bergh, J. C. J. M. (2000). "Themes, Approaches, and Differences with Environmental Economics". Tinbergen Institute Recuperado el 18-01-2010, Disponible en: http://www. tinbergen.nl 